\title{
Viscoelastic Particle Train Formation in Microfluidic Flows Using a Xanthan Gum Aqueous Solution
}

\author{
Anoshanth Jeyasountharan, Keshvad Shahrivar, Gaetano D’Avino, and Francesco Del Giudice* \\ Cite This: Anal. Chem. 2021, 93, 5503-5512 \\ Read Online
}

ABSTRACT: Viscoelastic polymer solutions have been widely employed as suspending liquids for a myriad of microfluidic applications including particle and cell focusing, sorting, and encapsulation. It has been recently shown that viscoelastic solutions can drive the formation of equally spaced particles called "particle trains" as a result of the viscoelasticity-mediated hydrodynamic interactions between adjacent particles. Despite their potential impact on applications such as droplet encapsulation and flow cytometry, only limited experimental studies on viscoelastic ordering are currently available. In this work, we demonstrate that a viscoelastic shear-thinning aqueous xanthan gum solution drives the self-assembly of particle trains on the centerline of a serpentine microfluidic device with a nearly circular cross section. After focusing, the flowing particles change their mutual distance and organize in aligned structures characterized by a preferential spacing, quantified in terms of distributions of the interparticle distance. We observe the occurrence of multi-particle strings, mainly doublets and triplets, that interrupt the continuity of the particle train. To account for the fluctuations in the number of flowing particles in the experimental window, we introduce the concept of local particle concentration, observing that an increase of the local particle concentration leads to an increase of doublets and triplets. We also demonstrate that using only a single tube to connect the sample to the microfluidic device results in a drastic reduction of doublets/triplets, thus leading to a more uniform particle train. Our findings establish the foundation for optimized applications such as deterministic droplet encapsulation in viscoelastic liquids and optimized flow cytometry.

\section{INTRODUCTION}

The migration of particles and cells transversally to the flow direction due to internal forces generated within the bulk of the flow has been widely exploited in microfluidics for applications ranging from flow focusing to cell separation. ${ }^{1}$ Such forces are not generated by external fields such as electric, acoustic, or magnetic field, but they are rather induced from within the suspending liquid by either inertial $l^{2,3}$ or viscoelastic ${ }^{4}$ forces. Inertial forces are relevant at generally large values of the volumetric flow rate, ${ }^{2,3}$ while viscoelastic forces are excited by adding few amounts of polymers to the suspending liquid ${ }^{4}$ in order to impart elastic properties to the matrix.

Inertial and viscoelastic forces have been extensively employed for the focusing and the separation of particles and cells on different positions along the channel cross section. ${ }^{1}$ For inertial flow, it was demonstrated that changes of the channel geometry led to a modification of the equilibrium positions of the flowing particles and cells. ${ }^{1-3}$ For viscoelastic fluids, instead, the flow properties of the suspending liquids were found to affect the equilibrium position of flowing particles and cells even in simple straight microfluidic channels. ${ }^{5}$ The size-dependent nature of both inertial and viscoelastic forces led to significant advancements in the separation of particles and cells in simple microfluidic geometries. $^{1,5}$

In addition to the well-studied phenomenon of the sizedependent transversal particle migration, nonlinear forces can be employed to achieve particle or cell ordering, that is, the formation of strings of equally spaced particles called "particle trains". ${ }^{-9}$ The ability to control interparticle spacing is extremely important to optimize encapsulation of particles or cells in droplets ${ }^{10-15}$ in order to avoid inclusion of multiple objects in the same droplet as well as the problem of empty droplets. ${ }^{11}$ Particle trains form at sufficiently large particle or cell concentrations as a consequence of the hydrodynamic interactions occurring between consecutive objects. ${ }^{16}$ The main bulk of existing literature has so far focused on the inertial ordering, meaning that inertial forces were employed to achieve particle ordering. ${ }^{17-19}$ Only very recently, an experimental evidence of self-assembly of particle trains in viscoelastic liquids was provided. ${ }^{8}$ Specifically, particles suspended in an aqueous hyaluronic acid solution displaying shear-thinning features (i.e., the shear viscosity decreases when increasing the flow rate in the microchannel) self-assembled in a nearly equally spaced structure at the centerline of a microfluidic channel. Viscoelastic ordering was also recently

Received: December 22, 2020

Accepted: March 1, 2021

Published: March 23, 2021 
observed by Liu et al., ${ }^{20}$ who designed a microfluidic device for on demand self-assembly of particles. Viscoelastic ordering has several advantages over inertial ordering, such as the fact that particles in viscoelastic liquids become equally spaced on the channel centerline where the shear rate is minimal and the velocity is maximum, at variance with the inertial ordering where particles are either ordered on multiple particle positions $^{19,21}$ or on a single line near the channel wall where large particle/cell rotation rates may result in blurred images in cytometry applications that employ line-scan-based interrogation. ${ }^{18}$ Moreover, viscoelastic ordering tends to occur in shear-thinning liquids, ${ }^{8}$ meaning that the velocity profile in a microchannel is more flat around the centerline compared to the parabolic one observed in Newtonian liquids. This results in smaller shear stresses acting on objects flowing around the tube centerline with obvious advantages when processing delicate cells. ${ }^{22}$ The capability of viscoelastic fluids to promote particle ordering has been also demonstrated through recent numerical simulations, ${ }^{23}$ where different values of the particle volume fraction led to different particle structures: at very low volume fractions, the particles did not significantly interact and the distribution of the interparticle distances did not change from the initial random one; at intermediate values of the particle concentration, the formation of nearly equally spaced trains was observed; at high volume fractions, strings of nearly touching particles were formed.

Despite the relevance and the potential impact in a variety of microfluidic applications, works on viscoelastic ordering are very limited, and many open questions still remain, as detailed in the following. The only two previously mentioned experimental studies ${ }^{8,20}$ considered aqueous solutions of hyaluronic acid as suspending fluids. It is still not clear whether different polymer solutions displaying shear-thinning features are able to drive formation of particle trains on the centerline. Furthermore, the hyaluronic acid solutions employed by Del Giudice et al. ${ }^{8}$ presented a large zero-shear viscosity, which can cause problems during particle or cell mixing. Previous studies, both numerical and experimental, failed to fully characterize the impact of doublets or triplets of attached particles on the formation of a stable train. Existing studies also failed to address the relevance of the fluctuation of particle concentration in microfluidic devices in the context of particle train formation: this phenomenon is widely present in microfluidics (see, for instance, Kahkeshani et al. ${ }^{21}$ ), but no characterization of its impact on particle ordering has been so far considered. Finally, the effect of the microfluidic connections between the sample reservoir and the microfluidic device on the continuity of the particle train remains unaddressed: this is important under an experimental point of view, as a simplified microfluidic setup for particle train generation can lead to a larger portfolio of applications in material science as well as in biomedical engineering.

Motivated by these observations, in the present work, we discovered that an aqueous solution of viscoelastic shearthinning xanthan gum $(\mathrm{XG})$ drove the formation of particle trains on the centerline of a hydrophilic serpentine microchannel. XG in water was chosen as the suspending liquid because of its strongly shear-thinning properties at relatively low mass concentrations of the polymer (meaning smaller zero-shear viscosity values), its low cost compared to other polymers such as hyaluronic acid, and because of the recent interest about using XG for different microfluidic applications. $^{24-26}$ Furthermore, we observed the formation of doublets and triplets of attached particles that disturbed the continuity of the train and we quantified their impact on the train formation. We also introduced the concept of local particle concentration in an attempt to clarify the effect of particle concentration on the train formation as if there were no concentration fluctuations. Finally, we showed that a simplified microfluidic device involving the least number of connections between the reservoir and the microfluidic device led to the formation of a more uniform train.

\section{THEORETICAL BACKGROUND}

The dynamics of a system of aligned particles suspended in a viscoelastic fluid and flowing in a microfluidic channel is a complex phenomenon. The hydrodynamic interactions between consecutive particles are mediated by fluid viscoelasticity, fluid dynamics conditions, and the characteristic dimensions of both the microfluidic device and the suspended particles. $^{23,28}$ A variation of the relative position between two consecutive particles due to hydrodynamic interactions is reflected along the whole particle system with a characteristic time depending on several parameters such as the flow rate, particle over channel size, solid concentration, and fluid rheology. ${ }^{23}$

In the simplest case of a pair of particles aligned at the channel centerline, previous numerical simulations reported that particles experienced either an attractive or a repulsive force depending on their initial distance and the Deborah number (definition of $\mathrm{De}$ is reported in the Results and Discussion section). For a Deborah number lower than a threshold $D e_{\text {cr }}$, two particles with an initial distance $s$ below a critical value $s_{\text {cr }}$ experience an attractive force, leading to the formation of a particle doublet (Figure 1a). On the contrary, particles with an initial distance of $s>s_{\mathrm{cr}}$ experience a repulsive force that pushes the two particles apart (Figure 1b). The value of the critical distance $s_{\mathrm{cr}}$ reduces for increasing values of the Deborah number (Figure $1 \mathrm{c}, \mathrm{d}$ ) and, for values larger than $D e_{\mathrm{cr}}$, the two particles experience only a repulsive force. The attraction-repulsion dynamics between two particles depends on the rheological properties as well: shear-thinning features in the suspending liquid led to a similar decreasing of the critical distance compared with near constant-viscosity liquids (Figure $1 \mathrm{e}, \mathrm{f}) .^{27,28}$

For a system made of three aligned particles (depicted in Figure 2), the dynamics becomes much more complex, now depending upon the two relative distances, say $s_{1}$ and $s_{2}$, and on the Deborah number $D e e^{27}$ For a Deborah number lower than the critical value $D e_{\text {cr }}$ and for at least one of the two relative distances lower than the critical value $s_{\mathrm{cr}}$, the trailing and middle particle form a pair while the leading one moves faster and becomes isolated (Figure $2 a-c$ ). Three isolated particles, on the contrary, are formed if the two interparticle distances $s_{1}$ and $s_{2}$ are both larger than the critical value $s_{\mathrm{cr}}$ (Figure $2 \mathrm{~d}$ ). The latter is the only possible scenario for a Deborah number higher than $D e_{\mathrm{cr}}$ (Figure 2e).

In a system of several aligned particles, the overall dynamic depends upon the mutual distance between all the interacting particles. Since hydrodynamic interactions lead to continuous variations of the distances between two consecutive particles over time, the final configuration of the particle system cannot be easily predicted. However, according to the existing numerical simulations, particle trains (strings of equally spaced particles) can be obtained when all the distances between consecutive particles in the aligned particle system are larger 

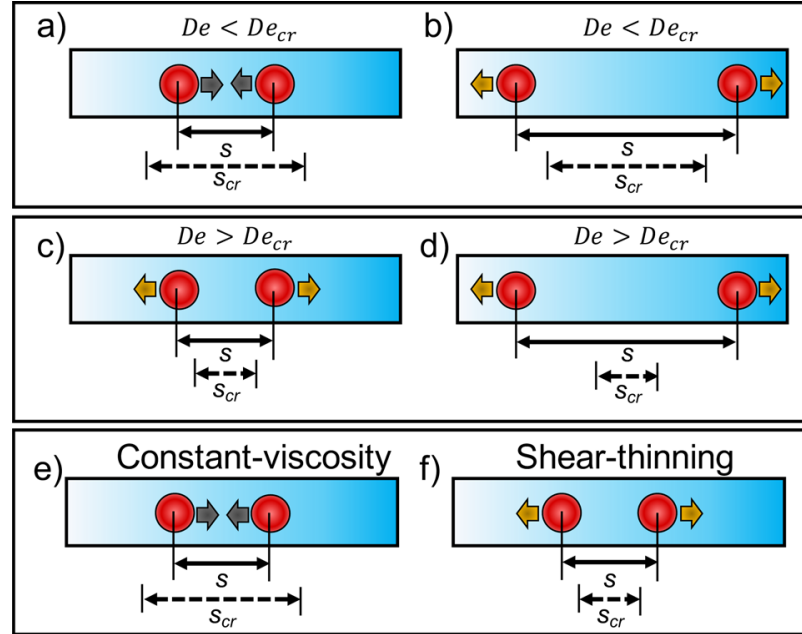

Figure 1. Schematic of attraction and repulsion dynamics between two flowing particles suspended in a viscoelastic liquid based on the numerical simulations of D'Avino et al. ${ }^{23,27}$ The value of the critical distance $s_{\mathrm{cr}}$ depends upon a number of parameters including the Deborah number $D e$ and the fluid rheological properties. (a) For a Deborah number lower than a threshold $D e<D e_{\mathrm{cr}}$, the particles at a distance of $s<s_{\mathrm{cr}}$ experience an attractive force, thus forming a doublet. (b) For $D e<D e_{\mathrm{cr}}$, adjacent particles at a distance of $s>s_{\mathrm{cr}}$ experience a repulsive force. (c) For $D e>D e_{\mathrm{cr}}$, the value of the critical distance $s_{\mathrm{cr}}$ becomes smaller than (a): particles at the same distance $s$ as in (a) now experience a repulsive force and are pushed further apart. (d) For $D e>D e_{\text {cr }}$, adjacent particles at a distance of $s>s_{\text {cr }}$ experience a repulsive force. (e) Particles suspended in a constantviscosity liquid (i.e., with negligible shear-thinning) with $s<s_{\mathrm{cr}}$ experience an attractive force resulting in the formation of a doublet. (f) When the suspending liquid presents shear-thinning features, the value of the critical distance $s_{\mathrm{cr}}$ becomes smaller than (e) and particles at the same distance $s$ as in (e) now experience a repulsive force.

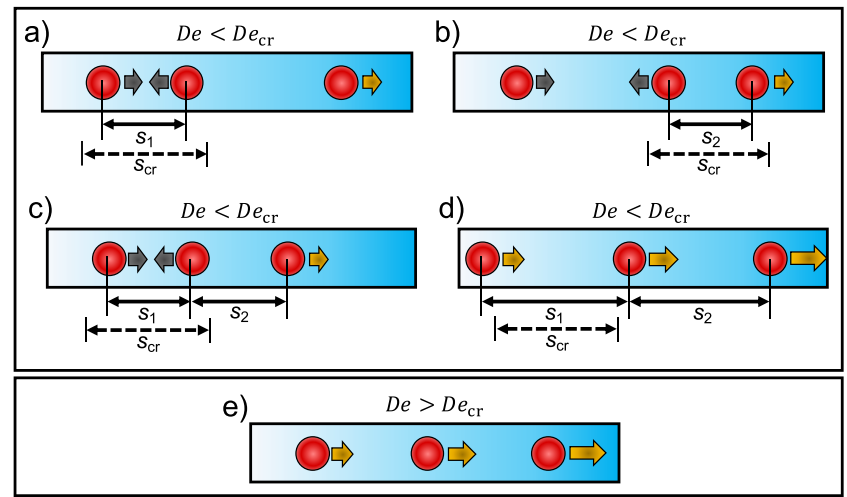

Figure 2. Schematic of attraction and repulsion dynamics between three flowing particles suspended in a viscoelastic liquid based on the numerical simulations of D'Avino et al. ${ }^{27}$ The value of the critical distance $s_{\mathrm{cr}}$ depends upon a number of parameters including the Deborah number $D e$ and the fluid rheological properties. The flow goes from the left to the right. $(\mathrm{a}-\mathrm{c})$ For a Deborah number lower than a threshold $D e<D e_{\text {cr }}$ if at least one interparticle distance $\left(s_{1}\right.$ or $s_{2}$ ) is lower than $s_{\mathrm{cr}}$, the trailing and middle particles form a doublet while the trailing one moves away, becoming isolated. (d) For $D e<$ $D e_{\mathrm{cr}}$ if both interparticle distances $\left(s_{1}\right.$ and $\left.s_{2}\right)$ are higher than $s_{\mathrm{cr}}$, the three particles separate and become isolated. (e) For $D e>D e_{\mathrm{cr}}$, the three particles separate regardless of the interparticle distances.

than the critical value $s_{\mathrm{cr}}$. It is also possible that the continuity of the particle train is broken by the occurrence of particle doublets formed because the initial distance between two particles is smaller than $s_{\mathrm{cr}}$.

\section{MATERIAL AND METHODS}

Sample Preparation and Characterization. The aqueous XG solution at 0.1 wt \% was prepared by dissolving Xanthomonas campestris (Sigma Aldrich UK) in deionized water. The solution was mixed using a magnetic stirrer for $12 \mathrm{~h}$ to allow full dissolution of the polymer.

The rheological measurements were conducted on a stresscontrolled rheometer (TA AR2000ex) with a truncated acrylic cone ( $60 \mathrm{~mm}$ diameter, $1^{\circ}$ angle) at a constant temperature ( $T$ $\left.=22{ }^{\circ} \mathrm{C}\right)$. A home-made solvent trap was used to prevent solvent evaporation of the XG solution during the rheological measurements.

The XG 0.1 wt \% exhibited strong shear-thinning features in the shear rate region $10^{-1}<\dot{\gamma}<10^{3} \mathrm{~s}^{-1}$ (Figure 3a). At shear
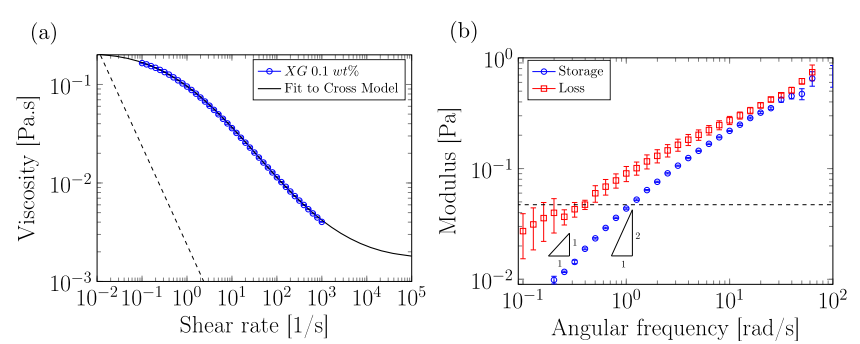

Figure 3. Rheological properties of XG $0.1 \mathrm{wt} \%$ in deionized water. (a) Shear viscosity $\eta$ as a function of shear rate $\dot{\gamma}$ in a shear rate range $10^{-1}<\dot{\gamma}<0.3 \mathrm{~s}^{-1}$. XG displayed shear thinning behavior above the critical shear rate $\dot{\gamma} \sim 1 \mathrm{~s}^{-1}$. (b) Storage $G^{\prime}$ and loss $G^{\prime \prime}$ moduli as functions of angular frequency $\omega$ for an imposed deformation $\gamma=5 \%$. Dashed lines in $(\mathrm{a}, \mathrm{b})$ show the minimum value $\eta_{\min }$ and $G_{\min }$, respectively, detectable by the rheometer due to torque limit: ${ }^{29}$ this is represented by $\eta_{\min }=2 T_{\min } / \pi R^{3} \dot{\gamma}(\mathrm{a})$ and $G_{\min }=2 T_{\min } /\left(\pi R^{3} \gamma\right)(\mathrm{b})$, where $T_{\min }=0.1 \mu \mathrm{N} \mathrm{m}$ is the minimum detectable torque, $R=30$ $\mathrm{mm}$ is the radius of the cone, and $\gamma$ is the strain amplitude.

rate values lower than $\dot{\gamma} \simeq 1 \mathrm{~s}^{-1}$, we observed an inflection of the viscosity as if the $\mathrm{XG}$ solution was attaining a constant zero-shear value; however, we were unable to explore experimentally the zero-shear region because the data appeared scattered, as affected by rheometrical edge effects. ${ }^{29}$

There is a significant bulk of literature (see, for instance, Song et al., ${ }^{30}$ Wyatt and Liberatore ${ }^{31}$ and references therein) where $\mathrm{XG}$ solutions in dilute and semi-dilute regimes were extensively characterized and their elastic properties were found to be significant. Elasticity in XG solutions is due to their stiff rod-like behavior in deionized water, similarly to aqueous solutions of rigid rods. ${ }^{30,31}$ Furthermore, the fact that in our experiments, rigid particles suspended in XG $0.1 \mathrm{wt} \%$ align on the centerline of the microfluidic device (see below) confirms that XG presents non-negligible elastic properties. ${ }^{22}$ In order to quantify the viscoelastic behavior of the $X G$ solution and to estimate the longest relaxation time $\lambda$, we also performed small angle oscillatory shear (SAOS) rheological measurements, where the storage modulus $G^{\prime}$ and the loss modulus $G^{\prime \prime}$ were evaluated as a function of angular frequency $\omega$ (Figure 3b). Despite observing a distinct viscoelastic behavior in the whole range of angular frequency investigated, we were unable to observe the "terminal region" at low angular frequencies, where the data are expected to scale with slopes 2 and 1 for $G^{\prime}$ and $G^{\prime \prime}$, respectively. ${ }^{32}$ Hence, we were not able to 
determine the longest relaxation time using standard SAOS measurements. A potential estimate for a relaxation time (not the longest one) could be performed as $\lambda^{*} \simeq 1 / \omega_{\mathrm{c}}=25 \mathrm{~ms}$, where $\omega_{\mathrm{c}}=40 \mathrm{rad} / \mathrm{s}$ is the frequency where $G^{\prime}=G^{\prime \prime}$ in Figure $3 \mathrm{~b}$. However, in agreement with previous works where the viscoelasticity was quantified using the longest relaxation time $\lambda$, we estimated this parameter by fitting the viscosity curve of Figure $3 \mathrm{a}$ with the Cross model

$$
\eta=\eta_{\infty}+\frac{\eta_{0}-\eta_{\infty}}{1+(\lambda \dot{\gamma})^{m}}
$$

where $\eta_{\infty}$ is the infinite shear viscosity, $\eta_{0}$ is the zero shear viscosity, $\lambda$ is the longest relaxation time, $\dot{\gamma}$ is the shear rate, and $m$ is the factor that modulates the transition between the constant region and the shear-thinning region. The fitted parameters are $\lambda=1.55 \mathrm{~s}, \eta_{0}=0.22 \mathrm{~Pa} \cdot \mathrm{s}, \eta_{\infty}=0.0018 \mathrm{~Pa} \cdot \mathrm{s}$, and $m=0.61$. Hereafter, we will employ a value of the longest relaxation time equal to $\lambda=1.55 \mathrm{~s}$ to discuss our results.

Polystyrene particles (Polysciences Inc.) with a diameter of $20 \pm 2 \mu \mathrm{m}$ were added to the $0.1 \mathrm{wt} \% \mathrm{XG}$ polymer solution at four different mass concentrations of $\phi=0.2 \mathrm{wt} \%, \phi=0.25 \mathrm{wt}$ $\%, \phi=0.3$ wt $\%$, and $\phi=0.4$ wt $\%$. The resulting suspension was mixed using a vortex mixer (Fisherbrand ZX3) to fully disperse the polystyrene particles in the XG polymer solution. The suspension was further put in an ultrasonic bath for $2 \mathrm{~min}$ to remove potential aggregates.

Microfluidic Apparatus and Particle Tracking. An inverted microscope (Zeiss Axiovert 135) was employed to analyze particle flow in a commercial hydrophilic glass $\mathrm{T}$ junction chip (Dolomite, Microfluidics) (see Figure 4a). According to the manufacturer, the device presented a nearly circular cross section having a height of $100 \mu \mathrm{m}$ and a width of

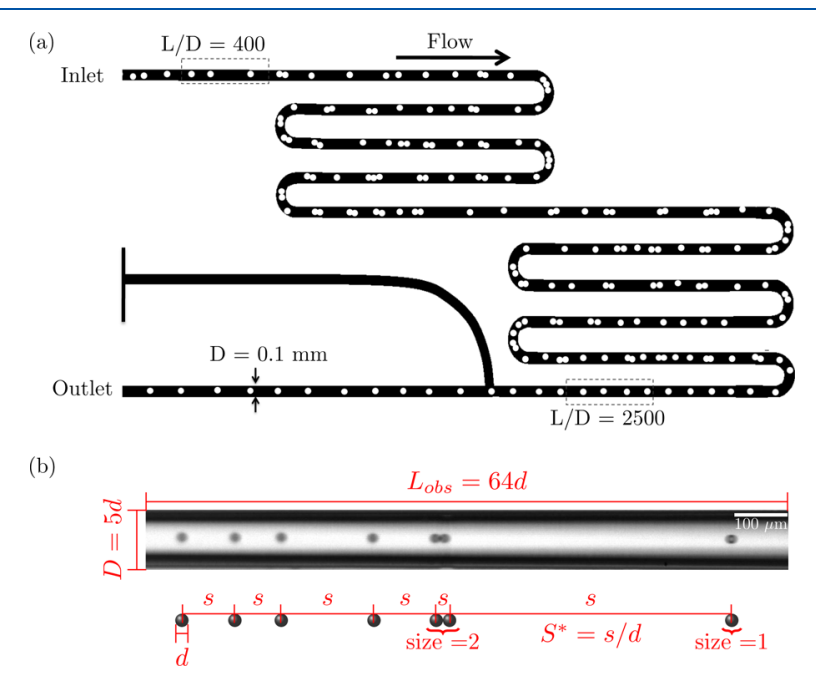

Figure 4. (a) Schematic representation of the microchannel with relevant dimensions. The internal diameter of the microchannel is $D=$ $0.1 \mathrm{~mm}$. Polystyrene particles with a diameter $d=20 \pm 2 \mu \mathrm{m}$ are observed at a distance from the channel inlet $L / D=400$ to verify the focusing and at $L / D=2500$ to analyze the self-assembling and evaluate the interparticle distances. Dimensions are not to scale. (b) The normalized distance between particles $S^{*}$ is determined by comparing the ratio between the center-to-center distances of adjacent particles $s$ with the particle diameter $d$, being $S^{*}=s / d$. Images thresholded to a binary image are used to determine the area of the particles, which in turn are used to determine the particle size. For instance, doublets were represented with a size of 2 . Scale bar is $100 \mu \mathrm{m}$.
$110 \mu \mathrm{m}$ with rounded corners. For the evaluation of the dimensionless Deborah number, we employed a value of the channel diameter equal to $D=100 \mu \mathrm{m}$. The T-junction chip was connected to a four-way Linear Connector (Dolomite, Microfluidics), which was connected to a $8 \mathrm{~mm}$ fluorinated ethylene propylene tube (Dolomite, Microfluidics) with an external diameter of $1.6 \mathrm{~mm}$ and an internal diameter of 0.25 $\mathrm{mm}$. Videos of flowing particles were captured with a fast camera (Photron, FASTCAM Mini UX50) at a frame rate of 2000-4000 frames per second depending on the imposed pressure drop. We selected a circular cross section in order to reduce as much as possible the migration of particles toward the channel walls, which is observed in square-shaped channels and enhanced for strongly shear-thinning fluids ${ }^{33}$ (as the one employed in the present work).

The suspension was pumped at various pressure drops $\Delta P$ with a pressure pump (Mitos p-pump) and the evolution of the flow rate $Q$ measured by the flow sensor (Dolomite Microfluidics) was monitored using the pressure pump computer software (Dolomite Microfluidics): the flow was considered to be stabilized once the value of $Q$ reached a steady state.

The following experimental protocol was employed in our investigation. First, a pressure drop corresponding to a flow rate of $Q=20 \mu \mathrm{L} / \mathrm{min}$ was imposed until the flow through the channel achieved a steady state. Then, the pressure drop $\Delta P$ was lowered to obtain a flow rate of $Q=5 \mu \mathrm{L} / \mathrm{min}$ to record the videos. Thereafter, $\Delta P$ was increased and videos were recorded at resulting flow rate values of $Q=7.5,10,15,20$, and $25 \mu \mathrm{L} / \mathrm{min}$. The experimental videos were then analyzed using a subroutine in order to derive the distance between consecutive particles (Figure $4 \mathrm{~b}$ ). The distance between adjacent particles was measured from center-to-center and divided by the particle diameter to obtain the normalized distance between particles, $S^{*}=s / d$, where $s$ is the center-tocenter distance between adjacent particles and $d$ is the particle diameter. Inter-particle distance histograms were then evaluated with (dimensionless) binning size equal to 1 and the two boundary ends were set to 1 and 64, which is the total length of the observation window (see Figure 4b).

\section{RESULTS AND DISCUSSION}

The results presented hereafter are discussed in terms of the Deborah number $D e$ that quantifies the degree of elasticity in response to the flow deformation. In agreement with previous works, ${ }^{22}$ we defined $\mathrm{De}$ as

$$
D e=\frac{4 \lambda Q}{\pi D^{3}}
$$

where $Q$ is the volumetric flow rate, $\lambda$ is the longest relaxation time, and $D$ is the diameter of the microfluidic channel. For Newtonian fluids, the relaxation time is zero (instant relaxation), ${ }^{32}$ and therefore, $D e=0$. For non-Newtonian fluids, the Deborah number is always positive.

Particle Focusing is the "Prelude" to Train Formation. Particle ordering, in inertial of viscoelastic flows, requires that adjacent flowing particles interact hydrodynamically with each other. ${ }^{7,8,19,27}$ This condition is fulfilled when the particle concentration is sufficiently large to make hydrodynamic interactions relevant between consecutive particles. Furthermore, longitudinal train formation requires that the particles are aligned along one streamline of the flow field. The experimental evidences provided by Del Giudice et al. ${ }^{8}$ 

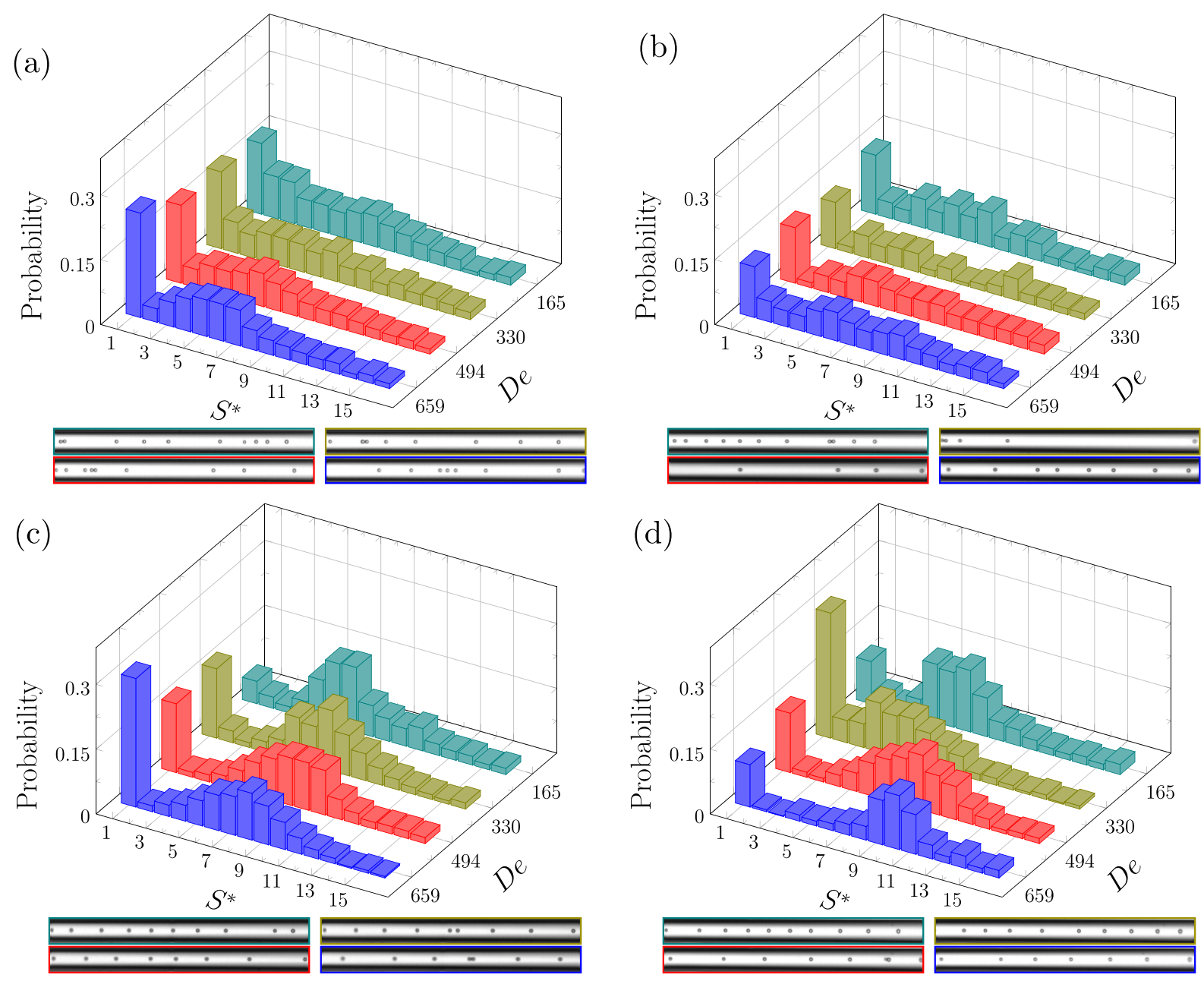

Figure 5. Histograms of the normalized distance $S^{*}=s / d$ for different Deborah numbers $D e$. (a,c) $L / D=400$ and $L / D=2500$, respectively, and for a particle bulk concentration of $\phi=0.2 \mathrm{wt} \%$. (b,d) $L / D=400$ and $L / D=2500$, respectively, and for $\phi=0.3 \mathrm{wt} \%$. For both $\phi$-values, no clear peak is observed in the distributions for $L / D=400$ except the one at $S^{*}=1$ denoting formation of doublets or triplets of touching particles. Particles exhibit some degree of ordering at $L / D=2500$. The interparticle distance depends upon the Deborah number De. Experimental snapshots at different $D e$ are shown below each figure with the same color code as the histograms.

suggested that self-assembly of particle trains is obtained only when the suspending liquid displays shear-thinning features; otherwise, adjacent particles will experience a substantial attractive force that would result in particle string formation rather than particle trains. In Newtonian liquids under inertialess conditions, particles do not focus or self-order, ${ }^{4}$ as also experimentally observed here (see Figure S1). To focus particles on the centerline of the microfluidic device in shearthinning liquids, Del Giudice et al. $^{22}$ demonstrated that the confinement ratio $\beta=d / D$ should be $\beta \geq 0.15$. For lower values of the confinement ratio, instead, particles were driven toward the lateral walls of the microfluidic device. ${ }^{22}$ As previously reported, in our experiments, we employed an aqueous XG 0.1 wt \% shear-thinning solution (Figure 3a) and particles with diameter $d=20 \mu \mathrm{m}$ flowing in a microfluidic channel with tube diameter of $D=100 \mu \mathrm{m}$, thus leading to a value for the confinement ratio $\beta=0.2$.

Polystyrene particles at two bulk concentrations of $\phi=0.2$ wt $\%$ and $\phi=0.3 \mathrm{wt} \%$ at a (dimensionless) distance from the channel inlet $L / D=400$, corresponding to $4 \mathrm{~cm}$ from the inlet of the microfluidic device, were perfectly focused on the channel centerline (snapshots in Figure 5a,b). However, they were not equally spaced, as confirmed by the $S^{*}=s / d$ distribution at different Deborah number values. The only peak in the probability function at $L / d=400$ was the one at $S^{*}$ $=1$, meaning that several particles were forming strings of particles in contact, mainly doublets (Figure S2). Doublet formation could be ascribed to the multiple connections existing between the reservoir and the microfluidic channel, all of them with different internal diameters. Intuitively, when a large concentration of particles experience a series of significant geometrical contractions and expansions, particle overcrowding might occur between consecutive connections, resulting in doublet formation, as also reported in previous experiments. ${ }^{34}$

As the distance between consecutive particles becomes smaller than a critical value, recent numerical simulations ${ }^{27,28}$ predicted that the particles experience an attractive force leading to doublet formation; a similar phenomenon has been observed experimentally by Del Giudice et al. ${ }^{8}$ We did not have direct optical access to the different connections; therefore, we could not make a clear conclusion on this point. However, the fact that the strings of observed particles tended to form mostly doublets seems to support our hypothesis rather than suggesting an intrinsic particle selfassembly dynamic influencing the whole train. Notice also that doublets or triplets formation involved only a maximum of 
$20 \%$ of the overall number of particles (Figure S2), while the remaining $80 \%$ was made of isolated particles.

These results showed that the particles focused relatively quickly $(L / D=400)$ even though no clear self-assembled structure could be observed (Figure 5a,b), apart from doublets or triplets (Figure S2). Since particle trains were observed at $L / D=2500$ as described in detail in the next section, this suggests that particle focusing is the "prelude" to particle ordering, in agreement with previous studies on viscoelastic ${ }^{8,20}$ and inertial ${ }^{16,35}$ ordering.

Train Formation in a Shear-Thinning XG Aqueous Solution. As described in the previous section, we observed particle focusing on the channel centerline at $L / D=400$, despite no clear ordering occurs (Figure 5a,b). When moving the observation point toward the outlet of the microfluidic device at $L / D=2500$ (corresponding to a distance from the channel inlet of $25 \mathrm{~cm}$ ), significant peaks in the distribution of $S^{*}$ appeared, regardless of the Deborah number (Figures $5 \mathrm{c}$, d and S3), denoting the existence of a preferential interparticle distance and the formation of a particle train (Movie S1). This is in good agreement with previous experimental results by Del Giudice et al. ${ }^{8}$ and Liu et al., ${ }^{20}$ and with recent numerical simulations by D'Avino and Maffettone. ${ }^{23}$

For a particle concentration of $\phi=0.2 \mathrm{wt} \%$, the peaks in the distributions at $L / D=2500$ (Figure $5 b$ ) were between $7<S^{*}$ $<8$ for all the Deborah numbers investigated, in agreement with the experimental results of Del Giudice et al. ${ }^{8}$ where the interparticle distance was found to be independent of $D e$. Since the average distance computed from the distributions at $L / D=$ 400 (Figure 5a) was about 7 particle diameters, we concluded that at $L / D=2500$ a good degree of ordering was obtained with a preferential distance consistent with the equilibrium one (i.e., the one expected if all the particles were equally spaced). Notice also that, for increasing values of $D e$, the preferential distance moved from $S^{*}=7$ to $S^{*}=8$ and, accordingly, the peak at $S^{*}=1$ increased (Movie S2). This behavior can be easily understood as, by increasing the number of particles that form doublets or triplets (those at $S^{*}=1$ ), the equilibrium distance of the particles in the train must increase. Taken together, these observations suggest that the equilibrium distributions at $L / D=2500$ are unaffected by the Deborah number and the slight deviations are due to the presence of a different amount of particles forming strings that, in turn, depends on the initial interparticle distances (see discussion below).

A different phenomenon was instead observed for the higher particle concentration $\phi=0.3$ wt $\%$, where a peak in the interparticle distance distribution was still clearly visible but at a value of $S^{*}$ that strongly depended on the Deborah number (Figure 5d). Specifically, the peak progressively moved at higher interparticle distances as $\mathrm{De}$ increases, in agreement with recent numerical simulations. ${ }^{23}$ A possible explanation for this behavior is that the particle train did not reach a stable regime yet, that is, by further increasing the distance from the channel inlet, the distributions in Figure 5d would change. As previously shown, ${ }^{23}$ the train dynamics is strongly affected by any interparticle distance variation that is more relevant at high particle concentrations as the particles are on the average closer, enhancing the hydrodynamic interactions. Another explanation is related to the concentration fluctuations as discussed later.

The distributions reported in Figure 5 also showed the existence of a relevant peak at $S^{*}=1$ denoting the formation of doublets and triplets of particles in contact. Such a peak, observed for all the investigated conditions, was also present relatively close to the channel inlet $(L / D=400)$, suggesting that this was a phenomenon related to the initial particle distribution rather than an effect of the particle ordering. As previously reported by numerical simulations, ${ }^{23,27}$ once two aligned particles achieved a distance smaller than a critical value, fluid viscoelasticity generated an attractive force leading to doublet formation. The particles forming the doublet could be hardly separated during the flow. This was confirmed by our experimental distributions where (except the peculiar case at $\phi$ $=0.2 \mathrm{wt} \%$ and $D e=165)$ the peak at $S^{*}=1$ remained nearly constant or increased from $L / D=400$ to $L / D=2500$. Needless to say, the formation of doublets/triplets is detrimental for particle ordering and should be avoided. In this regard, designing a microfluidic device with more complex geometries aimed at increasing the distance between close particles might help. An example of such a device was recently reported by Liu et al., ${ }^{20}$ where a complex serpentine-like microfluidic channel was developed to prevent doublet and triplet formation. An experimental apparatus with less microfluidic connections between the sample and the device would be beneficial to reduce the occurrence of doublet formation, which currently account for about $20 \%$ of the overall particles (Figure S2): this aspect is investigated later in this manuscript.

Effect of Local Particle Concentration on Particle Train Formation. As previously discussed, particle concentration has a relevant effect on the train dynamics. Particle concentration can be modified by simply increasing/reducing the number of particles in the stock solution, and this should result in a lower/higher preferential interparticle distance. In general, however, this is not true in microfluidic applications involving the flow of solid particulate because of the wellknown problem of concentration fluctuations. ${ }^{20,21}$ During experiments, despite the best effort to uniformly mix the stock solution, we clearly observed fluctuations in the number of particles per frame, with some frames being empty of particles (see for instance Movies S1 and S2). This means that the flowing particles may experience different ordering dynamics because the number of interacting particles over time is different. ${ }^{8,23}$ In other words, even for a fixed nominal bulk concentration, the actual concentration of particles observed in a frame may vary over time, thus making it not possible to understand the overall dynamic of the train.

In an attempt to clarify the effect of particle concentration on the train formation as if there were no concentration fluctuations, we introduced the concept of local particle concentration $\phi_{1}$. The local particle concentration accounted for the number of particles observed in a single frame and was defined as

$$
\phi_{1}=\frac{N_{\mathrm{p}} d}{L_{\mathrm{f}}}
$$

where $L_{\mathrm{f}}=64 d$ is the length of the observation window and $N_{\mathrm{p}}$ is the number of particles in the observation window for a specific frame. Notice that $\phi_{1}$ varied between 0 (no particle in the frame) and 1 (the aligned particles were in contact and formed a string with length equal to the observation window). Because of the fluctuations of particles flowing in the microfluidic device per unit time, the local concentration was in general different from the bulk concentration. From each 

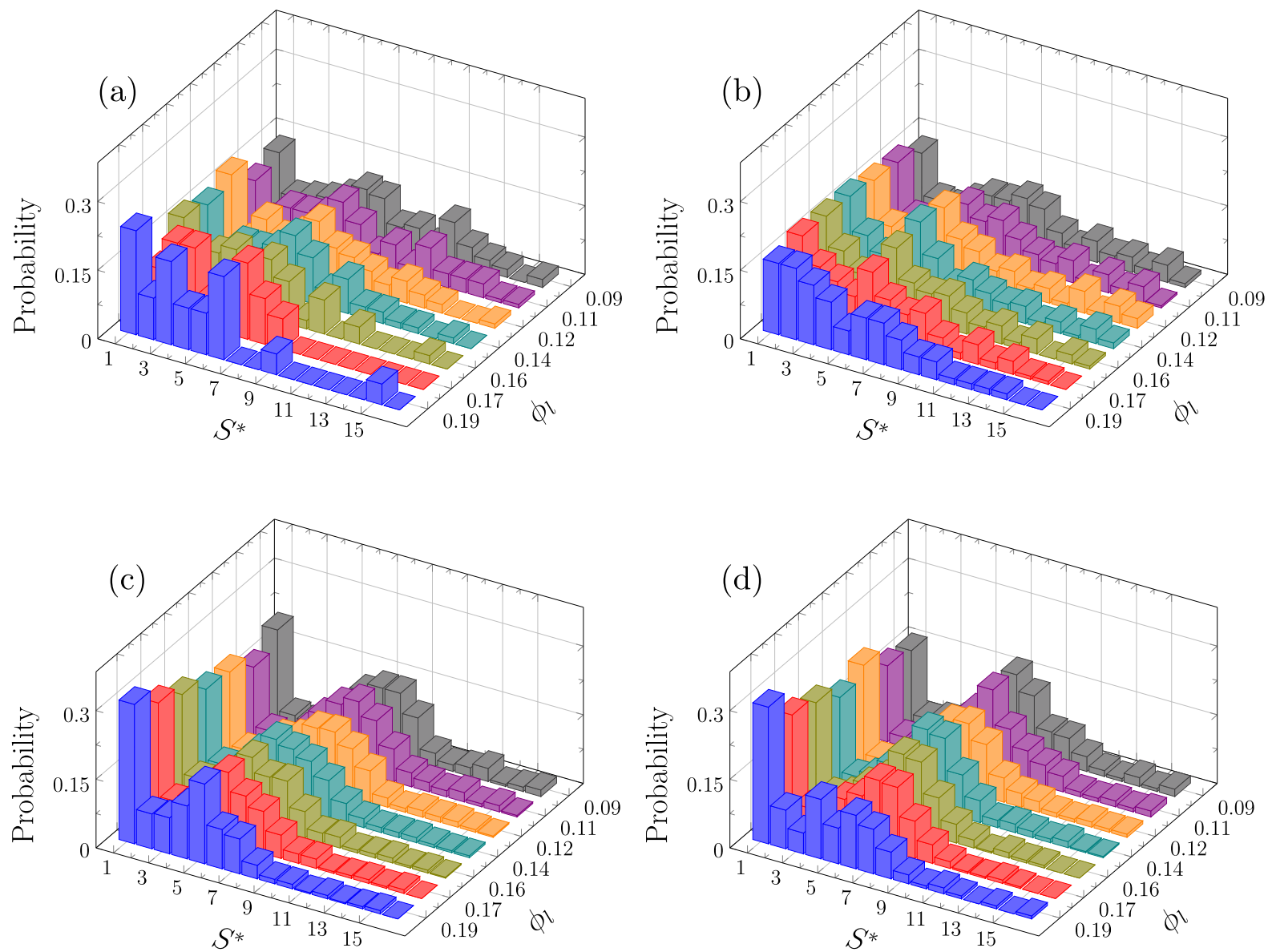

Figure 6. Histograms of the normalized distance $S^{*}=s / d$ for different local particle concentrations $\phi_{1}$ and for $D e=165(Q=5 \mu \mathrm{L} / \mathrm{min})$. (a,c) $L / D$ $=400$ and $L / D=2500$, respectively, and for a particle bulk concentration of $\phi=0.2 \mathrm{wt} \%$. (b,d) $L / D=400$ and $L / D=2500$, respectively, and for $\phi=0.3 \mathrm{wt} \%$. Higher $\phi_{1}$ increased the probability of doublet and triplet formation $\left(S^{*}=1\right)$.

video analyzed to produce the results in Figure 5, we identified all the independent frames having the same number of particles (i.e., the same local concentration). For each local concentration, we subsequently evaluated the average distribution of $S^{*}$ as a function of the Deborah number De (Figures 6, S4 and S5).

At $L / D=400$, we did not observe any clear peak in the distribution of inter-particle distances $S^{*}$ (Figure 6a,b), in agreement with our previous observations at different bulk concentrations (Figure 5a,b). When moving the observation point at $L / D=2500$, peaks in the interparticle distance at $S^{*}=$ 1 and $6<S^{*}<8$ appeared (Figure $6 \mathrm{c}, \mathrm{d}$ ). Interestingly, by increasing the local particle concentration, the peak at $S^{*}=1$ increased as well, suggesting that a higher particle concentration enhanced the formation of doublets and triplets rather than form trains with a smaller interparticle distance. These results, together with recent numerical simulations ${ }^{23}$ and other experiments, ${ }^{8}$ let us conclude that larger particle concentrations have to be avoided in the interest of a more uniform train formation. This means that there is a range of favorable particle concentrations for which a train can be formed: if the particle concentration is too low, then no train would be observable due to the weak hydrodynamic interactions between adjacent particles (in other words, the interparticle distances would remain unchanged from the initial distribution); on the other hand, if the particle concentration is too large, then particle doublets or triplets can form and disrupt the continuity of the train. This is also in line with similar observations on particle trains formed in inertial flows. ${ }^{19,21,36}$

We also studied the effect of the Deborah number on multiparticle string (i.e., doublets, triplets, etc.) formation as a function of the local particle concentration. The bars of Figure 7 represent the probability of forming multi-particle strings at

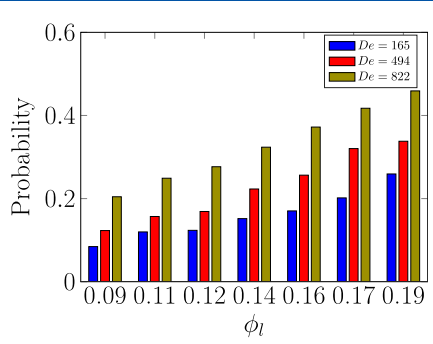

Figure 7. Probability of multi-particle string (doublets and triplets) formation as a function of local particle concentration $\phi_{1}$ and at different Deborah numbers. An increase of both the Deborah number and the local concentration promotes doublets and triplets formation.

different local concentrations for the three Deborah numbers $D e=165, D e=494$, and $D e=822$. As $D e$ increased, we observed that the probability of doublet and triplet formation increased for each specific local particle concentration. This means that when the concentration is fixed, an increase of the Deborah number results in a higher probability of doublet or triplet formation. For instance, at $\phi_{1}=0.09$ and at $D e=165$, we 
observed less than $10 \%$ of the particles forming doublets or triplets; an increase of Deborah to $D e=822$ resulted in around $20 \%$ of particles to form multi-string arrangements.

Taken together, our results demonstrate that large particle concentrations result in the formation of multi-strings of particles. This phenomenon is accentuated when increasing the Deborah number even for relatively low values of the local particle concentration.

In summary, the results so far confirmed that elastic fluids with shear-thinning features are the type of liquids required for the formation of particle trains. Previous numerical simulations by D'Avino et al. ${ }^{27}$ on the dynamics of particle pairs and triplets suggested that shear-thinning should help train formation. Indeed, they found that when the suspending liquid was shear-thinning, adjacent interacting particles would more likely experience a repulsive force; on the contrary, when the suspending liquid had negligible shear-thinning features, adjacent particles would experience an attractive force, thus leading to the formation of continuous strings of particles rather than particle trains. For a given suspending liquid, either shear-thinning or near constant-viscosity, the "likeliness" of experiencing attraction or repulsion was found to depend on the critical distance between the two adjacent particles. In other words, for a given suspending liquid, particles can form doublets or triplets when they become too close, that is, closer than the critical distance required to "activate" the attractive forces; otherwise, they would repel and likely form particle trains (Figure 1). Our observations are in line with previous findings, as we noticed self-assembly of particle trains together with the presence of doublets and triplets $\left(S^{*}=1\right)$, as reported in Figure 5c,d. As previously remarked, it is important to control the way adjacent particles interact with each other by properly designing the microfluidic channel.

Simplified Microfluidic Connections Result in a Drastic Reduction of Particle Doublets. In the previous paragraphs, we discussed the occurrence of multi-particle string formation and we argued that a better designed microfluidic device could potentially lead to a reduction of such particle strings, improving the continuity of the particle trains. We carried out an additional experiment where we simply removed the flow sensor from the pressure pump. Even though we could not directly read the volumetric flow rate, the advantage of this approach was a drastic reduction of connections between tubes having different internal diameters, thus reducing the occurrence of flow perturbations that could result in particle aggregation. Specifically, the configuration without the flow sensor presented a single connecting tube from the vial containing the particle suspension directly to the microfluidic device (Figure $8 \mathrm{a}$ ). This means that if particles were properly mixed and no aggregates were present, no multi-particle strings should form unless the critical distance between adjacent particles fell below the critical distance for particle attraction. ${ }^{23}$ We used this simplified experimental apparatus to study particle train formation in XG $0.1 \mathrm{wt} \%$ with a bulk particle concentration $\phi=0.4$ wt \% (Figure $8 \mathrm{~b}, \mathrm{c}$ ). We carried out experiments by imposing the pressure drop ranging in $200<$ $\Delta P<400$ mbar. An estimate of the flow rate has been done by measuring the velocity of aligned particles. Specifically, we run numerical simulations of the fluid without particles using the Cross model in eq 1 to derive the fluid velocity at the axis for an imposed flow rate. Then, we corrected such velocity to account for the presence of a confinement ${ }^{37}$ and verified at which flow rate the corrected fluid velocity matched the
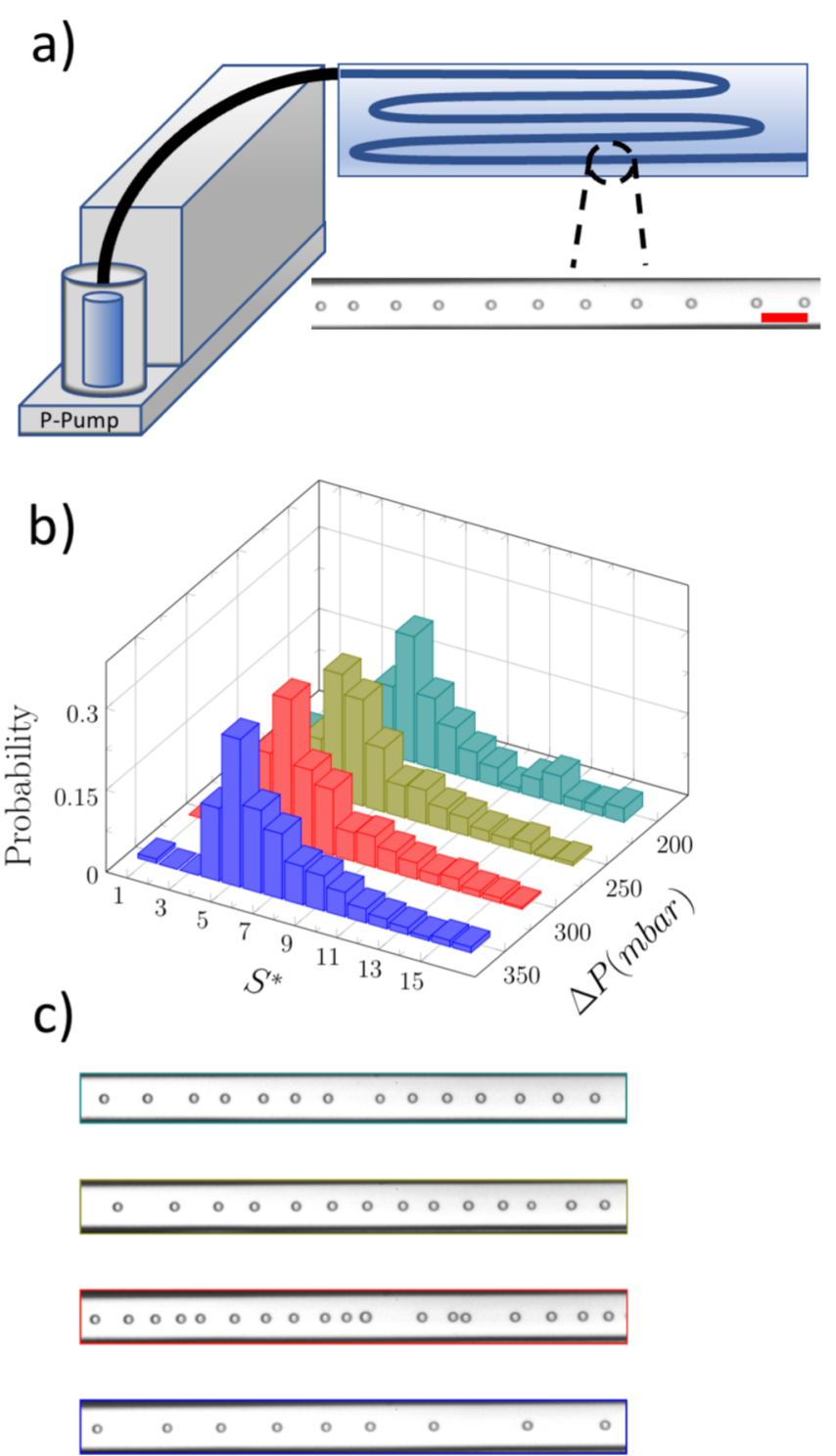

Figure 8. (a) Schematic of the simplified microfluidic configuration. Scale bar is $100 \mu \mathrm{m}$. (b)Histograms of the normalized distance $S^{*}=$ $s / d$ for different values of the pressure drop $\Delta P$ for $L / D=2500$ and for a particle bulk concentration of $\phi=0.4 \mathrm{wt} \%$. (c)Experimental snapshots at different $\Delta P$ are with the same color code as the histograms.

experimental one. The estimated flow rate range was $0.4<Q<$ $3.1 \mu \mathrm{L} / \mathrm{min}$ resulting in the Deborah number range of $10<D e$ $<80$.

In the whole range of imposed pressure drop, we observed a drastic reduction of particle doublets, with more uniform particle trains across the whole $\Delta P$ range (Movie S3). Notably, the pick in the $S^{*}$ distribution was independent of the imposed pressure drop $\Delta P$, meaning that they were independent of the Deborah number $D e$, in agreement with the results presented previously. Our results also confirmed that the multi-particle strings observed in Figure 5c,d were the result of the initial distribution of particles where doublets and triplets of particles were observed (Figure 5a,b). Most importantly, our results confirmed the fact that both channel design and experimental apparatus employed are essential to achieve more uniform particle trains in viscoelastic liquids. 


\section{CONCLUSIONS}

In this work, we demonstrated that a viscoelastic shearthinning aqueous XG solution 0.1 wt \% promoted the selfassembly of particle trains on the centerline of a serpentine microfluidic device. We showed that the particles focused relatively quickly $(L / D=400)$ even though no clear selfassembled structure could be observed. Particle train formation was found at $L / D=2500$, thus making particle focusing the "prelude" to particle ordering, in agreement with previous numerical simulations in viscoelastic liquids ${ }^{8,20}$ as well as previous studies in inertial flows. ${ }^{16,35} \mathrm{We}$ found that the preferential distance observed through the distributions of the interparticle distances depended on the particle concentration and the Deborah number. The latter suggested that a steadystate distribution was yet to be attained. The distributions were also characterized by a significant peak at a distance equal to the particle diameter, denoting the presence of several particles forming doublets or triplets. These were ascribed to the fact that adjacent particles with initial inter-particle distances below a critical value were subjected to viscoelasticity-mediated attractive forces and were hardly separated during the flow, in agreement with previous numerical simulations. ${ }^{29,28}$ Since the existence of these structures is detrimental for particle ordering, new channel designs need to be developed to avoid their formation. We also introduced the concept of local particle concentration to better understand the dynamic of train formation observed experimentally as if there were no particle concentration fluctuations. We observed that large local particle concentrations resulted in the formation of multistrings of particles. This phenomenon was accentuated when increasing the Deborah number, even for relatively low values of the particle concentration. Finally, we demonstrated that removing microfluidic connections between the reservoir and the device resulted in a more uniform particle train.

Future works are required to identify additional viscoelastic liquids where particle trains are observed. According to the existing literature, ${ }^{8,23}$ shear-thinning is a required feature to obtain particle trains, and our findings seem to suggest the same. Future studies regarding viscoelastic particle train formation in other polymer solutions such as polyethylene oxide or polyacrylamide (widely used for particle/cell manipulation $^{5}$ ) are required. Similarly, other studies where fluids with nearly constant-viscosity are employed as suspending liquids would provide additional insights into the particle train formation mechanism.

Our results are of interest in many microfluidic applications including flow cytometry, ${ }^{18}$ droplet encapsulation, ${ }^{10}$ and material science. ${ }^{38,39}$ Our results can also lead to novel optimized methodologies for flow cytometry and particle/cell separation by combining viscoelastic ordering and recent advances in both electro-viscoelastic migration ${ }^{40}$ and thermal buoyancy convection. ${ }^{41}$

\section{ASSOCIATED CONTENT}

\section{(s) Supporting Information}

The Supporting Information is available free of charge at https://pubs.acs.org/doi/10.1021/acs.analchem.0c05370.

Particles suspended in a Newtonian 25 wt \% glycerolwater solution do not focus on the centerline or selforder at both $L / D=400$ and $L / D=2500$; distribution of string size as a function of the Deborah number for $L /$ $D=400$ and $L / D=2500$; histogram of the normalized distance between the inter-particle distance $S^{*}=s / d$ for different Deborah numbers $D e$ for a particle bulk concentration $\phi=0.25$ wt $\%$ at $L / D=400$ and $L / D$ $=2500$; histograms of the normalized distance $S^{*}=s / d$ for different local particle concentrations $\phi_{1}$ and for $D e=$ 248; and histograms of the normalized distance $S^{*}=s / d$ for different local particle concentrations $\phi_{1}$ and for $D e=$ 659 (PDF)

Formation of particle trains at $L / D=2500$ for an imposed flow rate $Q=10 \mu \mathrm{L} / \mathrm{min}(\mathrm{MP} 4)$

Formation of particle trains at $L / D=2500$ for an imposed flow rate $Q=15 \mu \mathrm{L} / \mathrm{min}(\mathrm{MP} 4)$

Formation of particle trains at $L / D=2500$ using the experimental apparatus without a flow sensor at an imposed pressure drop $\Delta P=350$ mbar (MP4)

\section{AUTHOR INFORMATION}

\section{Corresponding Author}

Francesco Del Giudice - Systems and Process Engineering Centre, College of Engineering, Swansea University, Swansea SA1 8EN, U.K.; (1) orcid.org/0000-0002-9414-6937; Email: francesco.delgiudice@swansea.ac.uk

\section{Authors}

Anoshanth Jeyasountharan - Systems and Process Engineering Centre, College of Engineering, Swansea University, Swansea SA1 8EN, U.K.

Keshvad Shahrivar - Systems and Process Engineering Centre, College of Engineering, Swansea University, Swansea SA1 8EN, U.K.

Gaetano D'Avino - Dipartimento di Ingegneria Chimica, dei Materiali e della Produzione Industriale, Universitá degli Studi di Napoli Federico II, 80125 Naples, Italy

Complete contact information is available at: https://pubs.acs.org/10.1021/acs.analchem.0c05370

\section{Author Contributions}

A.J. and K.S. equally contributed to the work. F.D.G. designed the study. A.J. performed the experiments. K.S. wrote the subroutine code and analyzed the data. All the authors critically discussed the results. A.J. and K.S. drafted the manuscript. F.D.G. and G.D. wrote the final version of the manuscript. All the authors approved the final submission.

Notes

The authors declare no competing financial interest.

\section{ACKNOWLEDGMENTS}

A.J., K.S., and F.D.G. acknowledge support from EPSRC New Investigator Award (grant ref. no. EP/S036490/1).

\section{REFERENCES}

(1) Stoecklein, D.; Di Carlo, D. Anal. Chem. 2018, 91, 296-314.

(2) Di Carlo, D. Lab Chip 2009, 9, 3038-3046.

(3) Tang, W.; Zhu, S.; Jiang, D.; Zhu, L.; Yang, J.; Xiang, N. Lab Chip 2020, 20, 3485-3502.

(4) D’Avino, G.; Greco, F.; Maffettone, P. L. Annu. Rev. Fluid. Mech. 2017, 49, 341-360.

(5) Lu, X.; Liu, C.; Hu, G.; Xuan, X. J. Colloid Interface Sci. 2017, 500, 182-201.

(6) Segré, G.; Silberberg, A. Nature 1961, 189, 209-210.

(7) Matas, J.-P.; Glezer, V.; Guazzelli, É.; Morris, J. F. Phys. Fluids 2004, 16, 4192-4195. 
(8) Del Giudice, F.; D’Avino, G.; Greco, F.; Maffettone, P. L.; Shen, A. Q. Phys. Rev. Appl. 2018, 10, 064058.

(9) Xiang, N.; Dai, Q.; Ni, Z. Appl. Phys. Lett. 2016, 109, 134101.

(10) Edd, J. F.; Di Carlo, D.; Humphry, K. J.; Köster, S.; Irimia, D.; Weitz, D. A.; Toner, M. Lab Chip 2008, 8, 1262-1264.

(11) Abate, A. R.; Chen, C.-H.; Agresti, J. J.; Weitz, D. A. Lab Chip 2009, 9, 2628-2631.

(12) Kemna, E. W. M.; Schoeman, R. M.; Wolbers, F.; Vermes, I.; Weitz, D. A.; Van Den Berg, A. Lab Chip 2012, 12, 2881-2887.

(13) Ramji, R.; Wang, M.; Bhagat, A. A. S.; Tan Shao Weng, D.; Thakor, N. V.; Teck Lim, C.; Chen, C.-H. Biomicrofluidics 2014, 8, 034104.

(14) Schoeman, R. M.; Kemna, E. W. M.; Wolbers, F.; van den Berg, A. Electrophoresis 2014, 35, 385-392.

(15) Dietsche, C.; Mutlu, B. R.; Edd, J. F.; Koumoutsakos, P.; Toner, M. Microfluid. Nanofluidics 2019, 23, 83.

(16) Lee, W.; Amini, H.; Stone, H. A.; Di Carlo, D. Proc. Natl. Acad. Sci. U.S.A. 2010, 107, 22413-22418.

(17) Di Carlo, D.; Irimia, D.; Tompkins, R. G.; Toner, M. Proc. Natl. Acad. Sci. U.S.A. 2007, 104, 18892-18897.

(18) Goda, K.; Ayazi, A.; Gossett, D. R.; Sadasivam, J.; Lonappan, C. K.; Sollier, E.; Fard, A. M.; Hur, S. C.; Adam, J.; Murray, C.; Wang, C.; Brackbill, N.; Di Carlo, D.; Jalali, B. Proc. Natl. Acad. Sci. U.S.A. 2012, 109, 11630-11635.

(19) Humphry, K. J.; Kulkarni, P. M.; Weitz, D. A.; Morris, J. F.; Stone, H. A. Phys. Fluids 2010, 22, 081703.

(20) Liu, L.; Xu, H.; Xiu, H.; Xiang, N.; Ni, Z. Analyst 2020, 145, $5128-5133$.

(21) Kahkeshani, S.; Haddadi, H.; Di Carlo, D. J. Fluid Mech. 2015, 786, R3.

(22) Del Giudice, F.; Sathish, S.; D’Avino, G.; Shen, A. Q. Anal. Chem. 2017, 89, 13146-13159.

(23) D'Avino, G.; Maffettone, P. L. Meccanica 2020, 55, 317-330.

(24) Li, D.; Xuan, X. Microfluid. Nanofluidics 2019, 23, 54.

(25) Li, D.; Shao, X.; Bostwick, J. B.; Xuan, X. Microfluid. Nanofluidics 2019, 23, 125.

(26) Cho, M.; Hong, S. O.; Lee, S. H.; Hyun, K.; Kim, J. M. Micromachines 2019, 10, 535.

(27) D’Avino, G.; Hulsen, M.; Maffettone, P. Comput. Fluids 2013, $86,45-55$.

(28) D’Avino, G.; Maffettone, P. L. Microfluid. Nanofluidics 2019, 23, 82.

(29) Ewoldt, R. H.; Johnston, M. T.; Caretta, L. M. Complex Fluids in Biological Systems; Springer: New York, 2014; pp 207-241.

(30) Song, K.-W.; Kim, Y.-S.; Chang, G.-S. Fibers Polym. 2006, 7, 129-138.

(31) Wyatt, N. B.; Liberatore, M. W. J. Appl. Polym. Sci. 2009, 114, $4076-4084$

(32) Macosko, C. W. Rheology: Principles, Measurements, and Applications; Wiley-VCH, 1994.

(33) Del Giudice, F.; D’Avino, G.; Greco, F.; Netti, P. A.; Maffettone, P. L. Microfluid. Nanofluidics 2015, 19, 95-104.

(34) Dressaire, E.; Sauret, A. Soft Matter 2017, 13, 37-48.

(35) Yuan, D.; Zhao, Q.; Yan, S.; Tang, S.-Y.; Alici, G.; Zhang, J.; Li, W. Lab Chip 2018, 18, 551-567.

(36) Gao, Y.; Magaud, P.; Baldas, L.; Lafforgue, C.; Abbas, M.; Colin, S. Microfluid. Nanofluidics 2017, 21, 154.

(37) Higdon, J. J. L.; Muldowney, G. P. J. Fluid Mech. 1995, 298, 193-210.

(38) Arpin, K. A.; Mihi, A.; Johnson, H. T.; Baca, A. J.; Rogers, J. A.; Lewis, J. A.; Braun, P. V. Adv. Mater. 2010, 22, 1084-1101.

(39) Wang, P.; Casadei, F.; Shan, S.; Weaver, J. C.; Bertoldi, K. Phys. Rev. Lett. 2014, 113, 014301.

(40) Serhatlioglu, M.; Isiksacan, Z.; Özkan, M.; Tuncel, D.; Elbuken, C. Anal. Chem. 2020, 92, 6932-6940.

(41) Zhang, K.; Ren, Y.; Hou, L.; Jiang, T.; Jiang, H. Anal. Chem. 2020, 92, 2778-2786. 